\title{
Identification of Synchronous Generator model with Frequency Control Using Unscented Kalman Filter
}

\author{
Hossein Ghassempour Aghamolki, Zhixin Miao, Lingling Fan, Weiqing Jiang, Durgesh Manjure \\ Department of Electrical Engineering, University of South Florida, Tampa FL USA 33620. \\ Phone: 1(813)974-2031, Fax: 1(813)974-5250,Email: linglingfan@usf.edu.
}

\begin{abstract}
In this paper, phasor measurement unit (PMU) data-based synchronous generator model identification is carried out using unscented Kalman filter (UKF). The identification not only gives the model of a synchronous generator's swing dynamics, but also gives its turbine-governor model along with the primary and secondary frequency control block models. PMU measurements of active power and voltage magnitude, are treated as the inputs to the system while the measurements of voltage phasor angle, reactive power and frequency are treated as the outputs. UKF-based estimation is carried out to estimate the dynamic states and the parameters of the model. The estimated model is then built and excited with the injection of the inputs from the PMU measurements. The outputs of the estimation model and the outputs from the PMU measurements are compared. Case studies based on PMU measurements collected from a simulation model and real-world PMU data demonstrate the effectiveness of the proposed estimation scheme.

Keywords: Synchronous Generator, Phasor Measurement Unite (PMU), Unscented Kalman Filter (UKF), Frequency Control, Droop Regulation
\end{abstract}

\section{Introduction}

Supervisory Control and Data Acquisition (SCADA) systems use nonsynchronous data with low density sampling rate to monitor power systems. The measurements collected from SCADA can not capture the system dynamics. Phasor measurement units (PMUs) equipped with GPS antenna provide voltage and current phasors as well as frequency with a high density sampling rate up to $60 \mathrm{~Hz}$. PMU data can capture the system electromechanical dynamics. In this paper, PMU data will be used for synchronous generator parameter estimation.

Synchronous generator parameter estimation has been investigated in the literature. Based on the scope of estimation, some only investigated electrical state estimation (e.g. rotor angle and rotor speed)[1, 2], 
while others estimated both system states and generator parameters [3-6]. Based on estimation methods, there are at least two major systematic methods for parameter estimation: least squares estimation (LSE) [7-9] and Kalman filter-based estimation [10-14]. To use LSE for dynamic system parameter estimation, a window of data is required. On the other hand, Kalman filter-based estimation can carry out estimation procedures at each time step. Thus Kalman filter-based estimation can be used for online estimation. This is also one of the reasons why PMU data-based system identification opts for Kalman filter-based estimation $[10-14]$.

Kalman Filter was originally proposed for the linear systems. For nonlinear systems, there are two approaches to handle nonlinearity: extended Kalman filter (EKF) and unscented Kalman filter (UKF). In EKF, nonlinear systems are approximated by linear systems using linearization techniques. EKF was first applied by PNNL researchers in dynamic model identification using PMU data [5, 6, 10]. [5] focuses on parameter calibration for a simple generator dynamic model. [6] presents parameter calibration for a multimachine power system under varying fault locations, parameter errors and measurement noises. In [10], parameter calibration for a more complicated generator model which includes electromechanical dynamics, electromagnetic dynamics, exciter dynamics, voltage control blocks and power system stabilizer (PSS), was presented. EKF-based simple generator model estimation was also carried out in [11, 12]. Limitations of EKF method has also been investigated in [11].

In UKF, a nonlinear system model will not be linearized. The stochastic characteristic of a random variable is approximated by a set of sigma points. This technique is essentially Monto-Carlo simulation technique. Dynamic process of these sigma points will be computed based on the nonlinear estimation model. Statistic characteristics of the dynamic process will then be evaluated. UKF can overcome the limitation of the linearization process required by the EKF method. However, more computing effort is required due to the introduction of sigma points. In [13], UKF is applied for state estimation. Accuracy and convergence for both EKF and UKF are compared in [13]. This paper focuses on state estimation only. Parameter estimation was not discussed. In [14], UKF is applied to estimate the following parameters $E_{q}$, $x_{d}^{\prime}$ and $H$ along with states. A comparison of various Kalman filter methods is documented in [15].

The synchronous generator model identified in the aforementioned papers focuses on the generator electromechanical, electromagnetic and excitation system only. For example, a $4^{\text {th }}$ order transient generator estimation model is assumed in [15]; a subtransient generator estimation model is adopted in [10]. None has addressed frequency control system identification. The goal of this paper is to apply UKF for parameter and state estimation for a synchronous generator model consisting of electromechanical dynamics and frequency 
control. Contributions of this paper are summarized in the following paragraphs.

- Not only electromechanical dynamics related states and parameters, but also turbine-governor dynamics, primary and secondary frequency control parameters will be estimated. Estimation related to frequency control based on PMU data has not been seen in the literature.

Particularly, we will estimate the following parameters and states: inertia constant $H$, damping factor $D$, internal voltage $E_{q}$, transient reactance $x_{d}^{\prime}$, mechanical power input $P_{m}$, Droop regulation $R$, turbine-governor time constant $T_{r}$, and secondary frequency control integrator gain $K_{i}$.

Some parameters are difficult to estimate due to nonlinearity. Parameters conversion is adopted in this paper in order to make estimation easier.

- Event playback method [10] is used in this paper to validate the identified low-order model. For validation, estimated parameters will be used to create a dynamic simulation model. Then event playback will be used to inject the same inputs to the dynamic simulation model. The output signals from the simulation will be compared with the PMU measurements.

- Lastly, real-world PMU data-based identification will be used to demonstrate the effectiveness of the proposed estimation model.

This paper is organized as follows. Following a description of basics of UKF algorithm in Section 2, the implementation of UKF for dynamic generator model estimation is discussed in Section 3. Section 4 presents the validation process and case studies. Finally, Section 5 presents the conclusions of this paper.

\section{Basic Algorithm of UKF}

A continuous nonlinear dynamic system is represented by the following equations.

$$
\left\{\begin{array}{l}
\dot{x}(t)=\bar{f}[x(t), u(t), v(t)] \\
y(t)=h[x(t), u(t), v(t)]+w(t)
\end{array}\right.
$$

where, $x(t)$ is the vector of state variables, $y(t)$ is the vector of output variables, $u(t)$ is the vector of input variables, $v(t)$ is the non-additive process noise, and $\omega(t)$ is additive measurement noise. Considering the time step of $\Delta t,(1)$ can be written as (2) in the discrete time domain: 


$$
\left\{\begin{aligned}
x_{k} & =x_{k-1}+\bar{f}\left[x_{k-1}, u_{k-1}, v_{k-1}\right] \Delta t \\
& =f\left[x_{k-1}, u_{k-1}, v_{k-1}\right] \\
y_{k} & =h\left[x_{k}, u_{k}, v_{k}\right]+w_{k}
\end{aligned}\right.
$$

The state $x_{k}$ is considered as a random variable vector with an estimated mean value $\hat{x}_{k}$ and an estimated co-variance $P_{x_{k}}$. Vector $\psi_{k}$ is considered as a set of unknown model parameters. For simplification, $\psi_{k}$ can also be treated as states, where $\psi_{k+1}=\psi_{k}$. Then, the new state vector is $X_{k}=\left[\begin{array}{ll}x_{k}^{T} & \psi_{k}^{T}\end{array}\right]^{T}$. The state-space model in (2) is reformulated as:

$$
\left\{\begin{array}{l}
X_{k}=f\left[X_{k-1}, u_{k-1}, v_{k-1}\right] \\
y_{k}=h\left[X_{k}, u_{k}, v_{k}\right]+w_{k}
\end{array}\right.
$$

Kalman filter is a recursive estimation algorithm. At each time step, given the previous step's information, such as the mean of the state $\hat{X}_{k-1}$, the covariance of the state $P_{X_{k-1}}$, Kalman filter estimation will provide the statistic information of the current step, i.e., the mean of the state $\hat{X}_{k}$ and the covariance of the state $P_{X_{k}}$. Usually a prediction step estimates the information based on the dynamic model only, and a correction step corrects the information based on the current step's measurements. There are several references for UKF algorithm in literatures. For rest of this section, [16] is the reference for all UKF algorithm related equations.

Unscented Kalman filter (UKF) is a Monte-Carlo simulation method. A set of sigma points will be generated based on the given statistic information: mean and covariance of the states. Sigma points vectors will emulate the distribution of the random variable. The set of sigma points is denoted by $\chi^{i}$ and their mean value represented by $\hat{X}$ while their covariance represented by $P_{X}$. For $n$ number of state variables, a set of $2 n+1$ points are generated based on the columns of matrix $\sqrt{(n+\lambda) P_{X}}$. As shown below, at $k-1$ step, $2 n+1$ sigma points (vectors) are generated.

$$
\left\{\begin{array}{l}
\chi_{k-1}^{0}=\hat{X}_{k-1} \\
\chi_{k-1}^{i}=\hat{X}_{k-1}+\left[\sqrt{(n+\lambda) P_{X_{k-1}}}\right]_{i}, \quad i=1, \ldots, n \\
\chi_{k-1}^{i+n}=\hat{X}_{k-1}-\left[\sqrt{(n+\lambda) P_{X_{k-1}}}\right]_{i+n}, \quad i=1, \ldots, n
\end{array}\right.
$$

where $\lambda$ is a scaling parameter $\left(\lambda=\alpha^{2}(n+\kappa)-n\right), \alpha$ and $\kappa$ are positive constants. In the prediction step, 
prediction of the next step state will be carried out for all these sigma points. Based on the information of the sigma points of the next step, the mean and the covariance of the states will be computed. UKF will use weights to calculate the predicted mean and covariance. The associated weights are as follows.

$$
\left\{\begin{array}{l}
W_{m_{0}}=\frac{\lambda}{(n+\lambda)} \\
W_{c_{0}}=\frac{\lambda}{(n+\lambda)}+\left(1-\alpha^{2}+\beta\right) \\
W_{m_{i}}=\frac{1}{2(n+\lambda)}, \quad i=1, \ldots, 2 n \\
W_{c_{i}}=\frac{1}{2(n+\lambda)}, \quad i=1, \ldots, 2 n
\end{array}\right.
$$

where $\beta$ is a positive constant, $W_{m_{i}}$ is used to compute the mean value, and $W_{c_{i}}$ is used to compute the covariance matrix. $\alpha, \kappa$ and $\beta$ are the Kalman Filter parameters which can be used to tune the filter. Scaling parameter $\beta$ is used to incorporate prior knowledge of the distribution of $x(k)$ and for Gaussian distribution $\beta=2$ is optimal [hyun2011]. The scaling parameter $\alpha$ is a positive value used for an arbitrary small number to a minimum of higher order effects. To choose $\alpha$, two laws have to be take into accounts. First, for all choices of $\alpha$, the predicted covariance must be defined as a positive semidefinite. Second, The order of accuracy must be preserved for both the mean and covariance [17]. See [18] and [17] for more details regarding the effect of scaling parameter $\alpha$ on UKF tuning. In this case study, we have chosen $\alpha=10^{-4}, \beta=2$.

$\kappa$ is a scaling factor that controls how far away from the mean we want the points to be. A larger $\kappa$ will enable points further away from the mean be chosen, and a smaller $\kappa$ enables points nearer the mean to be chosen. Based on Equation (5), it can be also seen that when $\kappa$ gets larger, not only the sampled sigma points go further away from the mean, but also the weights of those samples get smaller. In another word, by choosing a larger $\kappa$, samples are chosen further and further away from mean with less weight assigned to those samples. Therefore, choosing appropriate $\kappa$ will reduce higher order errors of Tylor's series for predicting the mean and covariance of the states of the system. It is shown in [19] and [20], that if $x(k)$ is Gaussian, it is more appropriate to choose $\kappa$ in a way that $n+\kappa=3$. However, if the distribution of $x(k)$ is different, then we have to use different approach for choosing $\kappa$. Detailed discussion regarding UKF parameters can be found in [19-21]. In this case study, we choose $\kappa=0$ since we use total 7 sigma point and $n=3$.

The predicted sigma points at the $k$-th step $\left(\chi_{k}^{-}\right)$, the mean $\left(\hat{X}_{k}^{-}\right)$and the covariance $\left(P_{X_{k}}^{-}\right)$of the $k$-th 
step state are described in (6). Note the superscript ${ }^{-}$denotes a prior state or prediction.

$$
\left\{\begin{array}{l}
\chi_{k}^{i-}=f\left(\chi_{k-1}^{i}, u_{k-1}\right), i=0, \cdots, 2 n \\
\hat{X}_{k}^{-}=\sum_{i=0}^{2 n} W_{m_{i}} \chi_{k}^{i-} \\
P_{X_{k}}^{-}=\sum_{i=0}^{2 n} W_{c_{i}}\left(\chi_{k}^{i-}-\hat{X}_{k}^{-}\right)\left(\chi_{k}^{i-}-\hat{X}_{k}^{-}\right)^{T}
\end{array}\right.
$$

Subsequently, the predicted measurement sigma points $\gamma_{k}^{-}$can be generated by finding the predicted sigma points $\chi_{k}^{-}$through the measurement equation (7).

$$
\gamma_{k}^{i-}=h\left(\chi_{k}^{i-}, u_{k}\right), i=0, \cdots, 2 n
$$

Consequently, the weighted mean of the predicted measurement $\hat{y}_{k}^{-}$and the corresponding covariance matrix $P_{y_{k}}^{-}$as well as the cross-correlation matrix $P_{X_{k} y_{k}}^{-}$can be computed as shown in (8).

$$
\left\{\begin{array}{l}
\hat{y}_{k}^{-}=\sum_{i=0}^{2 n} W_{m i} \gamma_{k}^{i-} \\
P_{y_{k}}^{-}=\sum_{i=0}^{2 n} W_{c i}\left(\gamma_{k}^{i-}-\hat{y}_{k}^{-}\right)\left(\gamma_{k}^{i-}-\hat{y}_{k}^{-}\right)^{T}+R_{w} \\
P_{X_{k} y_{k}}^{-}=\sum_{i=0}^{2 n} W_{c i}\left(\chi_{k}^{i-}-\hat{X}_{k}^{-}\right)\left(\gamma_{k}^{i-}-\hat{y}_{k}^{-}\right)^{T}
\end{array}\right.
$$

where $R_{w}$ is the co-variance matrix related to the measurement noise $w$. In the correction step, UKF then updates the state using Kalman gain matrix $K_{k}$. The mean value $\hat{X}_{k}$ and co-variance matrix $P_{X_{k}}$ (superscript ${ }^{-}$denotes a prior state) are expressed as follows.

$$
\left\{\begin{array}{l}
K_{k}=P_{X_{k} y_{k}}^{-}\left(P_{y_{k}}^{-}\right)^{-1} \\
\hat{X}_{k}=\hat{X}_{k}^{-}+K_{k}\left[y_{k}-\hat{y}_{k}^{-}\right]^{T} \\
P_{X_{k}}=P_{X_{k}}^{-}-K_{k} P_{y_{k}}^{-} K_{k}^{T}
\end{array}\right.
$$

There are existing general Kalman filter Matlab toolboxes available. In this research, we use a general EKF/UKF toolbox developed by Helsinki University [22]. Specific models of PMU data-based synchronous generator estimation are described in the next section and coded in the toolbox. 


\section{Implementation of UKF for dynamic model parameter estimation}

In the proposed estimation model, a synchronous generator is considered as a constant voltage source behind an impedance. The electromechanical dynamics can be described by the following swing equation $[23]$.

$$
\left\{\begin{array}{l}
\frac{d \delta(t)}{d t}=\omega_{s}\left(\omega(t)-\omega_{0}\right) \\
\frac{d \omega(t)}{d t}=\frac{1}{2 H}\left(P_{m}-P_{g}(t)-D\left(\omega(t)-\omega_{0}\right)\right)
\end{array}\right.
$$

where $\delta(t), \omega(t), \omega_{0}$ and $\omega_{s}$ are the rotor angle in radius, rotor speed in pu, synchronous speed in pu and base speed (377 rad/s), respectively. Rewriting the dynamic equations in the discrete form, we will have:

$$
\left\{\begin{array}{l}
\delta_{k}=\delta_{k-1}+\left(\omega_{k-1}-\omega_{0}\right) \omega_{s} \Delta t \\
\omega_{k}=\omega_{k-1}+\frac{\Delta t}{2 H_{k-1}}\left(P_{m_{k-1}}-P_{g_{k-1}}-D_{k-1}\left(\omega_{k-1}-\omega_{0}\right)\right)
\end{array}\right.
$$

where $\Delta t$ is the sample period. The PMU measured data can be separated into two groups. One group is treated as the input signals to the dynamic model and the other group is treated as the outputs or measurements. A PMU provides five sets of data at a generator terminal bus: voltage magnitude $\left(V_{g}\right)$, voltage phase angle $(\theta)$, active power $\left(P_{g}\right)$, reactive power $\left(Q_{g}\right)$, and frequency $(f)$. The PMU data contains only the positive sequence in this application based on the assumption that the system is operated under balanced conditions. Based on the swing equation, the state vector of the system is defined as $x_{k}=\left[\begin{array}{ll}\delta_{k} & \omega_{k}\end{array}\right]^{T}$. If we treat the parameters (unknown mechanical power $P_{m}$, inertia constant $H$ and damping coefficient $D$ ) of the model as state variables, the augmented state vector will be $X_{k}=\left[\begin{array}{lllll}\delta_{k} & \omega_{k} & P_{m_{k}} & H_{k} & D_{k}\end{array}\right]^{T}$.

In this paper we will use terminal voltage magnitude $\left(V_{g}\right)$ and generator exported power $\left(P_{g}\right)$ as the input signals, the terminal voltage phasor angle $(\theta)$ together with the reactive power are treated as the output signals. The relationship between input and output signals can be written as follows.

$$
\left\{\begin{array}{l}
P_{g}=\frac{E_{q} V_{g}}{x_{d}^{\prime}} \sin (\delta-\theta) \\
Q_{g}=\frac{E_{q} V_{g} \cos (\delta-\theta)-V_{g}^{2}}{x_{d}^{\prime}}
\end{array}\right.
$$

From (12) we can write: 


$$
\left\{\begin{array}{l}
E_{q} V_{g} \sin (\delta-\theta)=P_{g} x_{d}^{\prime} \\
E_{q} V_{g} \cos (\delta-\theta)=\sqrt{\left(E_{q} V_{g}\right)^{2}-\left(P_{g} x_{d}^{\prime}\right)^{2}}
\end{array}\right.
$$

Based on (13), the output signals can be expressed by the input signals and state variables as follows.

$$
\left\{\begin{array}{l}
\theta_{g_{k}}=\delta_{k}-\tan ^{-1}\left(\frac{P_{g_{k}} x_{d_{k}}^{\prime}}{\sqrt{\left(E_{q_{k}} V_{g_{k}}\right)^{2}-\left(P_{g_{k}} x_{d_{k}}^{\prime}\right)^{2}}}\right) \\
Q_{g_{k}}=\frac{\sqrt{\left(E_{q_{k}} V_{g_{k}}\right)^{2}-\left(P_{g_{k}} x_{d_{k}}^{\prime}\right)^{2}}-V_{g_{k}}^{2}}{x_{d_{k}}^{\prime}} .
\end{array}\right.
$$

\subsection{Primary and Secondary Frequency Control}

In the case that a system loses its power balance, the primary frequency control of synchronous generators will adjust the mechanical power reference point to a new value based on the frequency deviation. The primary frequency control is composed of a proportional block with the deviation of frequency as the input, the change in the mechanical power reference as the output. If the frequency is below the nominal value, the mechanical power should be increased. Otherwise, the mechanical power will be decreased. The system frequency will achieve steady state after such compensation. However, the steady-state frequency will deviate from the nominal value. Secondary frequency control loop is then added in order to bring the frequency back to its normal value. The secondary frequency control is mainly composed of an integral block as shown in Fig. 1. The integral unit senses the deviation in frequency or rotor speed and adjusts the reference power $P_{c}$. This way, the generator will be able to adjust its power input responding to the system load change.

In Fig. 1, $R$ is the speed regulation constant, $\frac{1}{R}$ is named as the droop gain, and $T_{r}$ is the turbine-governor time constant. Additional dynamic equations are as follows after considering the frequency controls.

$$
\frac{d P_{m}}{d t}=\frac{1}{T_{r}}\left(P_{\text {ref }}-P_{c}-P_{m}-\frac{1}{R}\left(\omega-\omega_{0}\right)\right)
$$

Rewriting (15) in the discrete form, we have:

$$
P_{m_{k}}=P_{m_{k-1}}+\frac{\Delta t}{T_{r}}\left(P_{\mathrm{ref}}-P_{c_{k-1}}-P_{m_{k-1}}-\frac{1}{R}\left(\omega_{k-1}-\omega_{0}\right)\right)
$$

Similarly, the secondary frequency control can be written as:

$$
P_{c_{k}}=P_{c_{k-1}}+\left(\omega_{k-1}-\omega_{0}\right) K_{i_{k-1}} \Delta t .
$$


The state vector of the system is now defined as $x_{k}=\left[\begin{array}{lllll}\delta_{k} & \omega_{k} & P_{m_{k}} & P_{c_{k}}\end{array}\right]^{T}$. If we treat the parameters of the model as state variables, the augmented state vector will be $X_{k}=\left[\begin{array}{llllllll}\delta_{k} & \omega_{k} & P_{m_{k}} & P_{c_{k}} & R_{k} & K_{i_{k}} & H_{k} & D_{k}\end{array}\right]^{T}$. The complete generator estimation model is presented as follows.

$$
\left\{\begin{array}{l}
\delta_{k}=\delta_{k-1}+\left(\omega_{k-1}-\omega_{0}\right) \omega_{s} \Delta t \\
\omega_{k}=\omega_{k-1}+\frac{\Delta t}{2 H_{k-1}}\left(P_{m}-P_{g_{k-1}}+D_{k-1}\left(\omega_{k-1}-\omega_{0}\right)\right) \\
P_{m_{k}}=P_{m_{k-1}}+\frac{\Delta t}{T_{r_{k}}}\left(P_{\text {ref }}-P_{c_{k-1}}-P_{m_{k-1}}-\frac{1}{R_{k}}\left(\omega_{k-1}-\omega_{0}\right)\right) \\
P_{c_{k}}=P_{c_{k-1}}+\left(\omega_{k-1}-\omega_{0}\right) K_{i_{k-1}} \Delta t \\
R_{k}=R_{k-1} \\
K_{i_{k}}=K_{i_{k-1}} \\
H_{k}=H_{k-1} \\
D_{k}=D_{k-1} \\
T_{r_{k}}=T_{r_{k-1}}
\end{array}\right.
$$

The model will be adapted for PMU data-based estimation to enhance the convergence of the UKF algorithm. Some parameters will be converted to new parameters in the estimation process. The detailed conversion will be shown in Case Studies. Parameter conversion has also been adopted in the literature [14].

\section{Case Studies}

\subsection{Case Study Based On Simulation Data}

To generate PMU data for case study, time-domain simulation data is generated using Matlab/SimPowerSystems. Demos in Matlab/SimPowerSystems include a classic two-area nice-bus system [23] shown in Fig. 2. This system consists of four generators in two areas. Two tie-lines connect these two areas. At $t=1$ second, a three-phase low impedance fault occurs at Bus 101. After 0.2 second, the fault is cleared. A PMU is used to record power, voltage, and the frequency data from the terminal bus of Gen 1. The sampling interval is 0.01 second.

Three sets of data were recorded (shown in Fig. 3) and used to test UKF method. Each set of data represents a different model for Generator 1 in the simulation studies.

- Set 1: For benchmarking, the classical generator model ( a constant voltage source behind a transient reactance) is used in simulation. In this case, the dynamic model used in UKF is exactly the same as 
the simulation model.

- Set 2: A subtransient model which includes all damping winding dynamics is used to represent Generator 1 in simulation. In the estimation model, dynamics related to the flux and damping winding have all been ignored.

- Set 3: The power system stabilizer (PSS), automatic voltage regulator (AVR), and excitation system are added to the subtransient generator model in this simulation. Adding PSS, AVR, and excitation system adds transients to the internal voltage of generator $\left(E_{q}\right)$. In the estimation model, $E_{q}$ is assumed to be constant.

In addition, turbine-governor, primary and secondary frequency control models same as those in the estimation model have been considered in Matlab/SimPowerSystems-based simulation. The generator parameters can be found in Table 5. At least two initial guesses for each parameter will be used to demonstrate that UKF can converge to the same estimation.

Parameter Conversion. In the process of UKF tuning, we found that direct estimation of $R, T_{r}$ and $H$ leads to decreased rate of algorithm convergence. From (18), it can be anticipated that the state variables $\omega$ and $P_{m}$ are linearly related to $\frac{1}{2 H}, \frac{1}{R}$ and $\frac{1}{T_{r} R}$ respectively. Therefore, a small change in $R, T_{r}$ and $H$ results in big fluctuations in $P_{m}$ and $\omega$. In other words, the output measurements have insignificant sensitivity to the parameters $R, T_{r}$ and $H$, which makes the filter tuning very difficult. To address this issue, parameters $G=\frac{100}{2 H}, J=\frac{1}{T_{r} R}$ and $N=\frac{1}{T_{r}}$ will be estimated. With such changes, ignoring the damping coefficient $(D)$, (18) can be rewritten as (19).

$$
\left\{\begin{array}{l}
\delta_{k}=\delta_{k-1}+\left(\omega_{k-1}-\omega_{0}\right) \omega_{s} \Delta t \\
\omega_{k}=\omega_{k-1}+\frac{G_{k-1}}{100}\left(P_{m}-P_{g_{k-1}}\right) \Delta t \\
P_{m_{k}}=P_{m_{k-1}}+N_{k-1}\left(P_{\mathrm{ref}}-P_{c_{k-1}}-P_{m_{k-1}}\right) \Delta t-J_{k-1}\left(\omega_{k-1}-\omega_{0}\right) \Delta t \\
P_{c_{k}}=P_{c_{k-1}}+\left(\omega_{k-1}-\omega_{0}\right) K_{i_{k-1}} \Delta t \\
G_{K}=G_{k-1} \\
M_{K}=M_{k-1} \\
J_{K}=J_{k-1} \\
K_{i_{k}}=K_{i_{k-1}}
\end{array}\right.
$$


In the literature, $V, \theta, P$, and $Q$ of PMU data are used as an input-output for Kalman Filter [5, 11, 14]. However, in this paper, frequency control parameters are to be estimated. Based on our experience, without frequency measurements from the generator terminal bus, convergence of the estimation is problematic. Therefore, the frequency of generator terminal bus is recorded and used as an output. We also make a simplifying assumption that the frequency measured at the generator terminal bus is equivalent to the rotor speed $(\omega)$ in per unit. The output signals can be written by input signals and state variables in the discrete form as follows.

$$
\left\{\begin{array}{l}
\left.\theta_{g_{k}}=\delta_{k}-\tan ^{-1}\left(\frac{P_{g_{k}} x_{d_{k}}^{\prime}}{\sqrt{\left(E_{q_{k}} V_{g_{k}}\right)^{2}-\left(P_{g_{k}} x_{d_{k}}^{\prime}\right)^{2}}}\right)\right) \\
Q_{g_{k}}=\frac{\sqrt{\left(E_{q_{k}} V_{g_{k}}\right)^{2}-\left(P_{g_{k}} x_{d_{k}}^{\prime}\right)^{2}}-V_{g_{k}}^{2}}{x_{d_{k}}^{\prime}} \\
f_{k}=\omega_{k}
\end{array}\right.
$$

For this estimation model, $E_{q}, P_{\text {ref }}, x_{d}^{\prime}$ are assumed known. In the UKF algorithm, $P$ is the co-variance matrix of the state variables, $X_{0}$ is the initial guess of the augmented state vector and $P_{0}$ is the initial guess for the co-variance matrix $P$. Estimation accuracy is not sensitive to the initial guess of parameters or state variables. Initial guess for covariance matrix $\left(P_{0}\right)$ will influence the convergence rate. Therefore, fine tuning of $P_{0}$ is needed. $Q$ is the co-variance matrix of the process noise and kept constant for all three sets of data. Table 1 shows the initial guess for $X_{0}$ and $P_{0}$ as well as diagonal elements of process noise matrix $Q$. $R_{w}$ is the covariance matrix of the output measurement noise $\left(R_{w}=\operatorname{diag}\left(\begin{array}{lll}10^{-15} & 10^{-15} & 10^{-15}\end{array}\right)\right)$.

Figs. 4-7 present the estimation of states compared to the states from the simulation model. As it can be seen, because the same classic generator model is used for both estimation and simulation model, the rotor angle estimation matches the simulated rotor angle exactly for Set 1 scenario. In both Set 2 and Set 3 , subtransient generator model is used in simulation while classic generator model is employed in estimation. Therefore, there is discrepancy between the rotor angles from the estimation and from the simulation, though the dynamic trends match each other well. Detailed discussion about such discrepancy can be found in [11]. Figs. 8-11 show the estimation and simulation result for inertia constant, turbine-governor time constant, droop regulation and the secondary frequency control gain respectively. It is found that even for a complicated generator model equipped with PSS and AVR, UKF can estimate all parameters and state variables with good accuracy.

Measurement Noises. In previous scenarios, the measurement errors were assumed to be normal distribution and very small (variance: $10^{-15}$ ). In order to show the effect of noise of normal distribution on the proposed 
method, three different simulation scenarios were carried out with adding $1 \%, 2 \%$ and $5 \%$ Gaussian noises to the Set 3 of the recorded data. The estimation results are compared to the previous parameters estimation. Table 2 presents the results for those scenarios. As it would be expected, It can be seen from the table that estimation error increases exponentially with increasing variance of the measurement noise. Although the error of the estimation increases with respect to the increasing level of measurement noise, the results of the proposed method still shows acceptable accuracy for the most of its applications.

Model Validation. In the validation step, estimated parameters are used to build a low order generator dynamic simulation model as shown in Fig. 1. Then, event playback proposed in $[4,24]$ is used to validate the estimation model. During event play back, hybrid dynamic simulation injects the inputs (measured PMU data) to the low-order dynamic simulation model, output from the model will be captured and compared with the actual measurements.

In the previous sections, although UKF is used to estimate parameters, some parameters such as $x_{d}^{\prime}$ and $E_{q}$ are assumed to be known. Moreover, all the generator model needs to have damping ratio to stabilize the system. Therefore, in this section, UKF method is adjusted to estimate all the parameters of the model. In another word, transient reactance $\left(x_{d}^{\prime}\right)$, generator internal voltage $\left(E_{q}\right)$ and generator damping ratio $(D)$ are added to the parameters which have to be estimated by UKF method. Thus, the augmented state vector will be $X_{k}=\left[\begin{array}{lllllllllll}\delta_{k} & \omega_{k} & P_{m_{k}} & P_{c_{k}} & G_{k} & J_{k} & M_{k} & K_{i_{k}} & x_{d_{k}}^{\prime} & E_{q_{k}} & D_{k}\end{array}\right]^{T}$. The PMU data are presented in Fig. 3. Kalman filter's parameter estimation are demonstrated in Figs. 12 and 13.

Estimated parameters have been used to build a continuous dynamic model of the generator in Matlab/Simulink. Then, input data (active power and voltage magnitude) are fed into the model to generate outputs. Frequency, reactive power, along with voltage phase angle are compared with the data measurements. Fig. 14 shows the result of validation. Three sets of models are constructed, one with all parameters included, the second one without considering secondary frequency control (without $K_{i}$ ) and the third one without considering any frequency control system (without $R$ and $T_{r}$ ). As demonstrated in Fig. 14, considering the frequency control systems in the estimation model will greatly improve the match of the outputs and the PMU data.

\subsection{Case Study Based on Real-World PMU Data}

In this section, UKF method is applied on the PMU data from an anonymous busbar of the MISO system to estimate parameters of a generator dynamic model. In the real world applications, the only data available is limited to PMU measurements. Equivalent dynamic models are sought. Therefore, it can be anticipated 
that for the real-world application, all the parameters of the generator are unknown and have to be estimated by the UKF method. The augmented state vector will be $X_{k}=\left[\begin{array}{llllllllllll}\delta_{k} & \omega_{k} & P_{m_{k}} & P_{c_{k}} & G_{k} & J_{k} & M_{k} & K_{i_{k}} & x_{d_{k}}^{\prime} & E_{q_{k}} & D_{k}\end{array}\right]^{T}$. The initial guess of the state variables $X_{0}$ and its covariance matrix $P_{0}$ as well as the covariance matrix for the processing noise are listed in Table 3.

Fig. 15 shows the PMU data of 40 seconds. The set of data was recorded by PMUs after a generator trip event. The data contain significant noises. Besides, PMUs save the data with a $30 \mathrm{~Hz}$ sampling rate. Data starting from 12 seconds to 40 seconds are used for estimation. Note in the figures follows, the starting time is 12 seconds. Experiments show that $30 \mathrm{~Hz}$ sampling rate does not yield satisfactory performance of UKF. This finding concurs with the findings documented in [15] that measurements interpolation is needed to improve the performance of Kalman filters. Our experiments show that the real data have to be interpolated to $100 \mathrm{~Hz}$ for the UKF method to converge. Figs. 16 and 17 present the estimation processes. Table 4 documents the final parameters estimation results.

In the next step, the low-order model with estimated parameters was built in Matlab/Simulink. Event playback is used to inject voltage magnitude and active power as inputs. The outputs from the estimated model and the output PMU measurements (frequency, voltage phase angle and reactive power) are compared. Fig. 18 shows the validation results. It is observed that despite the high level of noise and unknown dynamic system model structure, comparison of the PMU data with the validation model outputs shows a good degree of match. The real-world PMU data case study demonstrates the feasibility of the proposed estimation model in identifying a generator model.

\section{Conclusion}

$\mathrm{UKF}$ is implemented in this paper to estimate dynamic states and parameters of a low-order synchronous generator model with both primary and secondary frequency control systems. The proposed method uses voltage magnitude and active power measurements as inputs, voltage angle, reactive power and frequency as outputs. Inertia constant, damping coefficient, turbine-governor time constant, droop regulation as well as secondary frequency control gain will all be estimated. Both simulation data and real-world PMU data are used for case studies. In this research, various techniques are implemented to improve UKF algorithm for the this application. The techniques include: (i) parameter conversion to increase parameter detection sensitivity from the measurements; (ii) measurements interpolating to have a higher sampling rate to improve UKF convergence. In the validation step, a low-order dynamic simulation model is constructed with the estimated parameters. Input data are fed into the model to generate output data. The generated output 
data will then be compared with the outputs from the measurements.

The case studies demonstrate the feasibility of the proposed UKF estimation approach for system identification using PMU data. Through the proposed estimation method, a complex generator model can be emulated using a low-order generator with frequency controls. The case study on the real-world PMU data demonstrates the capability of the proposed UKF on identifying an equivalent generator model.

\section{Acknowledgement}

This work was supported partially through DOE/MISO grant "System Identification using PMU Data." The first author would like to acknowledge the help from Vahid Disfani and Javad Khazaei.

\section{References}

[1] P. Yang, Z. Tan, A. Wiesel, A. Nehora, Power system state estimation using pmus with imperfect synchronization, Power Systems, IEEE Transactions on 28 (4) (2013) 4162-4172.

[2] K. Schneider, Z. Huang, B. Yang, M. Hauer, Y. Nieplocha, Dynamic state estimation utilizing high performance computing methods, in: Power Systems Conference and Exposition, 2009. PSCE '09. IEEE/PES, 2009, pp. 1-6.

[3] Z. Huang, P. Du, D. Kosterev, B. Yang, Application of extended kalman filter techniques for dynamic model parameter calibration, in: Power Energy Society General Meeting, 2009. PES '09. IEEE, 2009, pp. 1-8.

[4] Z. Huang, D. Kosterev, R. Guttromson, T. Nguyen, Model validation with hybrid dynamic simulation, in: Power Engineering Society General Meeting, 2006. IEEE, 2006, pp. 1-6.

[5] Z. Huang, K. Schneider, J. Nieplocha, Feasibility studies of applying kalman filter techniques to power system dynamic state estimation, in: Power Engineering Conference, 2007. IPEC 2007. International, 2007, pp. 376-382.

[6] K. Kalsi, Y. Sun, Z. Huang, P. Du, R. Diao, K. Anderson, Y. Li, B. Lee, Calibrating multi-machine power system parameters with the extended kalman filter, in: Power and Energy Society General Meeting, 2011 IEEE, 2011, pp. 1-8.

[7] M. Burth, G. C. Verghese, M. Velez-Reyes, Subset selection for improved parameter estimation in on-line identification of a synchronous generator, Power Systems, IEEE Transactions on 14 (1) (1999) 218-225.

[8] M. Karrari, O. Malik, Identification of physical parameters of a synchronous generator from online measurements, Energy Conversion, IEEE Transactions on 19 (2) (2004) 407-415.

[9] C. Lee, O. T. Tan, A weighted-least-squares parameter estimator for synchronous machines, Power Apparatus and Systems, IEEE Transactions on 96 (1) (1977) 97-101.

[10] Z. Huang, P. Du, D. Kosterev, S. Yang, Generator dynamic model validation and parameter calibration using phasor measurements at the point of connection, Power Systems, IEEE Transactions on 28 (2) (2013) 1939-1949.

[11] L. Fan, Y. Wehbe, Extended kalman filtering based real-time dynamic state and parameter estimation using pmu data, Electric Power Systems Research 103 (2013) 168-177.

[12] E. Ghahremani, I. Kamwa, Dynamic state estimation in power system by applying the extended kalman filter with unknown inputs to phasor measurements, Power Systems, IEEE Transactions on 26 (4) (2011) 2556-2566. 
[13] E. Ghahremani, I. Kamwa, Online state estimation of a synchronous generator using unscented kalman filter from phasor measurements units, Energy Conversion, IEEE Transactions on 26 (4) (2011) 1099-1108.

[14] M. Ariff, B. Pal, A. Singh, Estimating dynamic model parameters for adaptive protection and control in power system, IEEE Trans. Power Syst. PP (99) (2014) 1-11.

[15] N. Zhou, D. Meng, Z. Huang, G. Welch, Dynamic state estimation of a synchronous machine using pmu data: A comparative study, Smart Grid, IEEE Transactions on PP (99) (2014) 1-1.

[16] S. S. Haykin, S. S. Haykin, S. S. Haykin, Kalman filtering and neural networks, Wiley Online Library, 2001.

[17] S. Julier, The scaled unscented transformation, in: American Control Conference, 2002. Proceedings of the 2002, Vol. 6, 2002, pp. 4555-4559 vol.6.

[18] J. J. Hyun, L. Hyung-Chul, Analysis of scaling parameters of the batch unscented transformation for precision orbit determination using satellite laser ranging data, Journal of Astronomy and Space Sciences 28 (3) (2011) 183-192.

[19] S. Julier, J. Uhlmann, H. Durrant-Whyte, A new approach for filtering nonlinear systems, in: American Control Conference, Proceedings of the 1995, Vol. 3, 1995, pp. 1628-1632 vol.3.

[20] S. Julier, J. Uhlmann, H. Durrant-Whyte, A new method for the nonlinear transformation of means and covariances in filters and estimators, Automatic Control, IEEE Transactions on 45 (3) (2000) 477-482.

[21] S. J. Julier, J. K. Uhlmann, New extension of the kalman filter to nonlinear systems, in: AeroSense'97, International Society for Optics and Photonics, 1997, pp. 182-193.

[22] H. Jouni, S. Simo, Optimal filtering with kalman filters and smoothers. manual for matlab toolbox ekf/ukf, Helsinki University of Technology, Department of Biomedical Engineering and Computational Science.

[23] P. Kundur, N. J. Balu, M. G. Lauby, Power system stability and control, Vol. 7, McGraw-hill New York, 1994.

[24] D. Kosterev, Hydro turbine-governor model validation in pacific northwest, Power Systems, IEEE Transactions on 19 (2) (2004) 1144-1149.

\section{Table of Tables}

1. Initial values for Three Parameters Estimation for a generator with Primary frequency control.

2. Effect of measurement noise on parameter estimation.

3. Initial state variables and covariance matrices for real-world PMU Data.

4. Parameters Estimation for Real-world PMU Data.

5. Generator parameters in Simpower simulations. 
Table 1: Initial values for Three Parameters Estimation for a generator with Primary frequency control

\begin{tabular}{|c|c|c|c|c|c|c|c|}
\hline$X_{0}$ & All Sets & $P_{0}$ & Set 1 & Set 2 & Set 3 & $Q$ & All Sets \\
\hline \hline$\delta$ & 0 & $P_{11}$ & 0.1 & 0.1 & 0.1 & $Q_{11}$ & $10^{-5}$ \\
\hline$\omega$ & 1 & $P_{22}$ & $10^{-5}$ & $1 e^{-5}$ & $10^{-5}$ & $Q_{22}$ & $10^{-11}$ \\
\hline$P_{m}$ & 0.8 & $P_{33}$ & 0.1 & 0.1 & 0.1 & $Q_{33}$ & $10^{-9}$ \\
\hline$P_{c}$ & 0 & $P_{44}$ & $10^{-5}$ & $1 e^{-5}$ & $1 e^{-5}$ & $Q_{44}$ & $10^{-9}$ \\
\hline$G$ & 1 & $P_{55}$ & $10^{-4}$ & $1 e^{-4}$ & 80 & $Q_{55}$ & $10^{-4}$ \\
\hline$J$ & 10 & $P_{66}$ & 240 & 35 & 76 & $Q_{66}$ & $10^{-12}$ \\
\hline$N$ & 1 & $P_{77}$ & 6.3 & 3.4 & 10 & $Q_{77}$ & $10^{-6}$ \\
\hline$K_{i}$ & 10 & $P_{88}$ & 77 & 64 & 20 & $Q_{88}$ & $10^{-4}$ \\
\hline
\end{tabular}

Table 2: Effect of measurement noise on parameters estimation

\begin{tabular}{|l|c|c|c|c|c|c|c|c|}
\hline \multirow{2}{*}{ scenarios } & \multicolumn{2}{|c|}{$\mathrm{H}$} & \multicolumn{2}{c|}{ Droop } & \multicolumn{2}{c|}{$T_{r}$} & \multicolumn{2}{c|}{$K_{i}$} \\
\cline { 2 - 9 } & Estimated & Error (\%) & Estimated & Error (\%) & Estimated & Error (\%) & Estimated & Error (\%) \\
\hline Simulation & 6.5 & 0 & 0.2 & 0 & 0.1 & 0 & 50 & 0 \\
\hline $0 \%$ error & 6.4773 & 0.35 & 0.1992 & 0.40 & 0.1005 & 0.52 & 50.0127 & 0.02 \\
\hline $1 \%$ error & 6.4763 & 0.37 & 0.1992 & 0.40 & 0.1007 & 0.67 & 50.0391 & 0.08 \\
\hline $2 \%$ error & 6.4411 & 0.91 & 0.1989 & 0.56 & 0.0990 & 1.00 & 49.8697 & 0.26 \\
\hline $5 \%$ error & 6.8068 & 4.72 & 0.1948 & 2.61 & 0.1059 & 5.93 & 50.4820 & 0.97 \\
\hline
\end{tabular}

Table 3: Initial state variables and covariance matrices for real-world PMU Data

\begin{tabular}{|c|c|c|c|c|c|}
\hline$X_{0}$ & values & $P_{0}$ & values & $Q$ & values \\
\hline \hline$\delta$ & 0 & $P_{1,1}$ & 0.1 & $Q_{1,1}$ & $1 e^{-5}$ \\
\hline$\omega$ & 1 & $P_{2,2}$ & 0.1 & $Q_{2,2}$ & $1 e^{-9}$ \\
\hline$P_{m}$ & 0.8 & $P_{3,3}$ & $1 e^{-4}$ & $Q_{3,3}$ & $1 e^{-9}$ \\
\hline$P_{c}$ & 0.8 & $P_{4,4}$ & $1 e^{-2}$ & $Q_{4,4}$ & $1 e^{-9}$ \\
\hline$G$ & 15 & $P_{5,5}$ & 0.1 & $Q_{5,5}$ & $1 e^{-8}$ \\
\hline$J$ & 50 & $P_{6,6}$ & 1000 & $Q_{6,6}$ & $1 e^{-5}$ \\
\hline$N$ & 20 & $P_{7,7}$ & 0.1 & $Q_{7,7}$ & $1 e^{-6}$ \\
\hline$k_{i}$ & 10 & $P_{8,8}$ & 0.71 & $Q_{8,8}$ & $1 e^{-12}$ \\
\hline$D$ & 0 & $P_{9,9}$ & $1 e^{-4}$ & $Q_{9,9}$ & $1 e^{-8}$ \\
\hline$x_{d}^{\prime}$ & 0.1 & $P_{10,10}$ & $1 e^{-3}$ & $Q_{10,10}$ & $1 e^{-7}$ \\
\hline$E_{q}$ & 0.9 & $P_{11,11}$ & $1 e^{-4}$ & $Q_{11,11}$ & $1 e^{-12}$ \\
\hline
\end{tabular}

Table 4: Parameters Estimation for Real-world PMU Data

\begin{tabular}{|c|c|c|c|c|c|c|}
\hline$H$ & $R$ & $T_{r}$ & $K_{i}$ & $D$ & $x_{d}^{\prime}$ & $E_{q}$ \\
\hline 20.18 & 0.0176 & 0.073 & 1.3448 & 0.031 & 0.1947 & 1.0538 \\
\hline
\end{tabular}


Table 5: Generator parameters in Simpower simulations

\begin{tabular}{|c|c|c|c|}
\hline Parameters & Set 1 & Set 2 & Set 3 \\
\hline \hline$E_{q}(\mathrm{pu})$ & 1.0567 & 1.8537 & 1.8537 \\
\hline$x_{d}^{\prime}(\mathrm{pu})$ & 0.3 & 0.3 & 0.3 \\
\hline$x_{d}^{\prime \prime}(\mathrm{pu})$ & - & 0.25 & 0.25 \\
\hline$x_{q}^{\prime}(\mathrm{pu})$ & - & 0.55 & 0.55 \\
\hline$x_{q}^{\prime \prime}(\mathrm{pu})$ & - & 0.25 & 0.25 \\
\hline$T_{d o}^{\prime}(\mathrm{pu})$ & - & 8 & 8 \\
\hline$T_{d o}^{\prime \prime}(\mathrm{pu})$ & - & 0.03 & 0.03 \\
\hline$T_{q o}^{\prime}(\mathrm{pu})$ & - & 0.4 & 0.4 \\
\hline$T_{q o}{ }^{\prime \prime}(\mathrm{pu})$ & - & 0.05 & 0.05 \\
\hline$P_{\text {ref }}(\mathrm{pu})$ & 0.778 & 0.779 & 0.779 \\
\hline$H(\mathrm{pu} . \mathrm{sec})$. & 6.5 & 6.5 & 6.5 \\
\hline$R(\mathrm{pu})$ & 0.1 & 0.1 & 0.1 \\
\hline$T_{r}(\mathrm{sec})$. & 0.1 & 0.1 & 0.1 \\
\hline$K_{i}$ & 50 & 50 & 50 \\
\hline AVR Gain $\left(K_{a}\right)$ & - & - & 200 \\
\hline AVR $T_{a}$ & - & - & 0.001 \\
\hline Exciter $K_{e}$ & - & - & 1 \\
\hline Exciter $T_{e}$ & - & - & 0 \\
\hline PSS $K_{p}$ & - & - & 30 \\
\hline PSS $T_{w}$ & - & - & 10 \\
\hline PSS lead lag 1 $T_{\text {num }}$ & - & - & 0.05 \\
\hline PSS lead lag 1 $T_{\text {den }}$ & - & - & 0.02 \\
\hline PSS lead lag $2 T_{\text {num }}$ & - & - & 3 \\
\hline PSS lead lag $2 T_{\text {den }}$ & - & - & 5.4 \\
\hline & & & \\
\hline
\end{tabular}




\section{Table of Figures}

1. Synchronous generator model including primary and secondary frequency controls.

2. The Study System.

3. Three sets of Matlab/SimPowerSystems simulation generated PMU measured data. Blue solid lines: Set 1; Read dashed lines: Set 2; Black dot-Dashed: Set 3.

4. Rotor angle estimation and simulation results. Top: Set 1, Middle: Set 2, Bottom: Set 3.

5. Rotor Speed estimation and simulation results. Top: Set 1, Middle: Set 2, Bottom: Set 3.

6. Mechanical Power estimation and simulation results. Top: Set 1, Middle: Set 2, Bottom: Set 3.

7. Mechanical Power reference point estimation and simulation results. Top: Set 1, Middle: Set 2, Bottom: Set 3.

8. Inertia constant estimation and simulation results. Top: Set 1, Middle: Set 2, Bottom: Set 3.

9. Turbine time constant estimation and simulation results. Top: Set 1, Middle: Set 2, Bottom: Set 3 .

10. Droop regulation estimation and simulation results. Top: Set 1, Middle: Set 2, Bottom: Set 3 .

11. Secondary Frequency Control Integrator Gain estimation and simulation results. Top: Set 1, Middle: Set 2, Bottom: Set 3 .

12. Set 3 All Generator Parameters estimation and simulation results.

13. Set 3 All Generator Parameters estimation and simulation results (continued).

14. Set 3 Model validation and outputs comparison. Top: Rotor Speed $\omega$, Middle: Voltage Phase Angle $\theta$, Bottom: Reactive Power $Q_{g}$.

15. Real-world PMU data obtained from a PMU installed at a $500 \mathrm{kV}$ substation. The data has been scaled to pu values, where $S_{b}=1000 \mathrm{MW}, V_{b}=500 \mathrm{kV}$.

16. Real-world system parameter estimation results.

17. Real-world parameter estimation results. Top: $x_{d}^{\prime}$, Middle: $E_{q}$ Estimation, Bottom: Damping Estimation.

18. Comparison of the real-world measurements and the simplified simulation model outputs. Top: Rotor Speed $\omega$, Mid: Voltage Phase Angle $\theta$, Bottom: Reactive Power $Q_{g}$. 


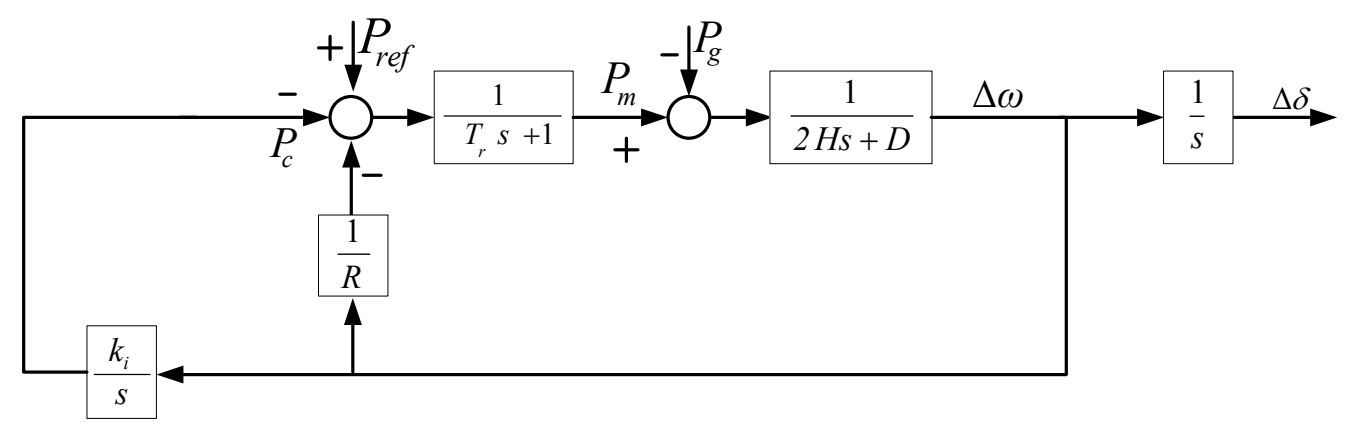

Figure 1: Synchronous generator model including primary and secondary frequency controls.

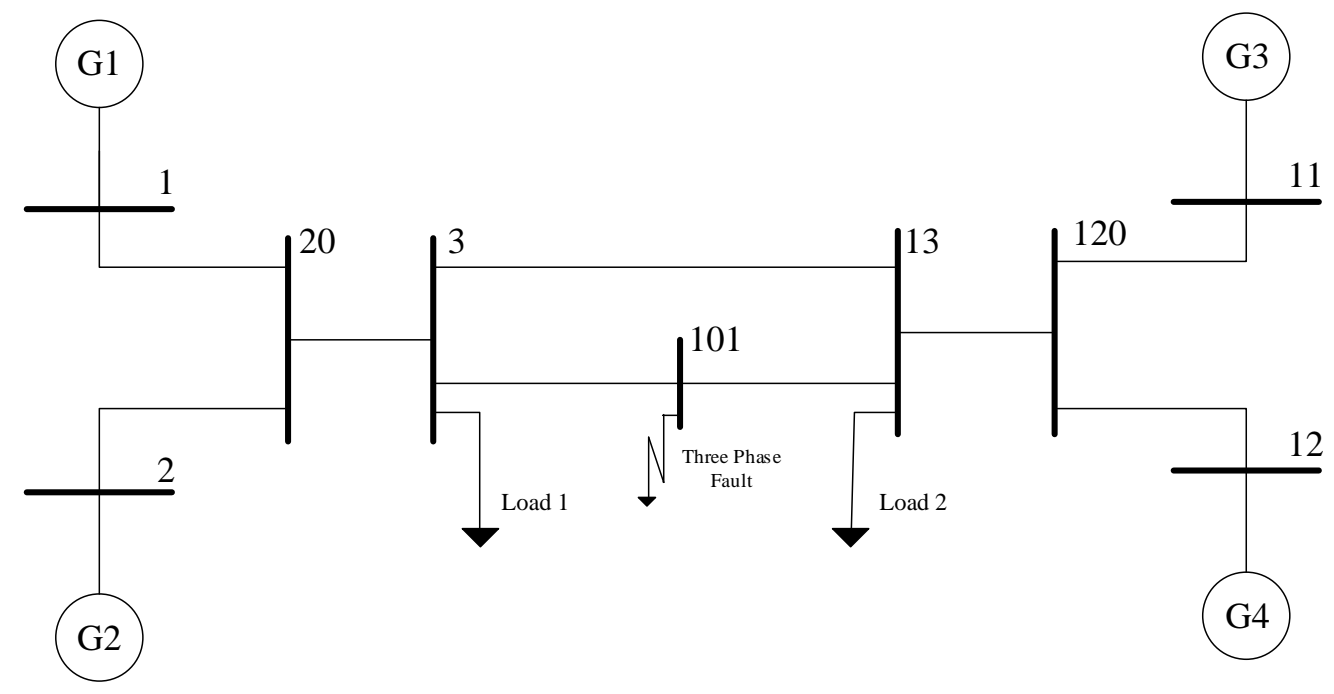

Figure 2: The Study System. 

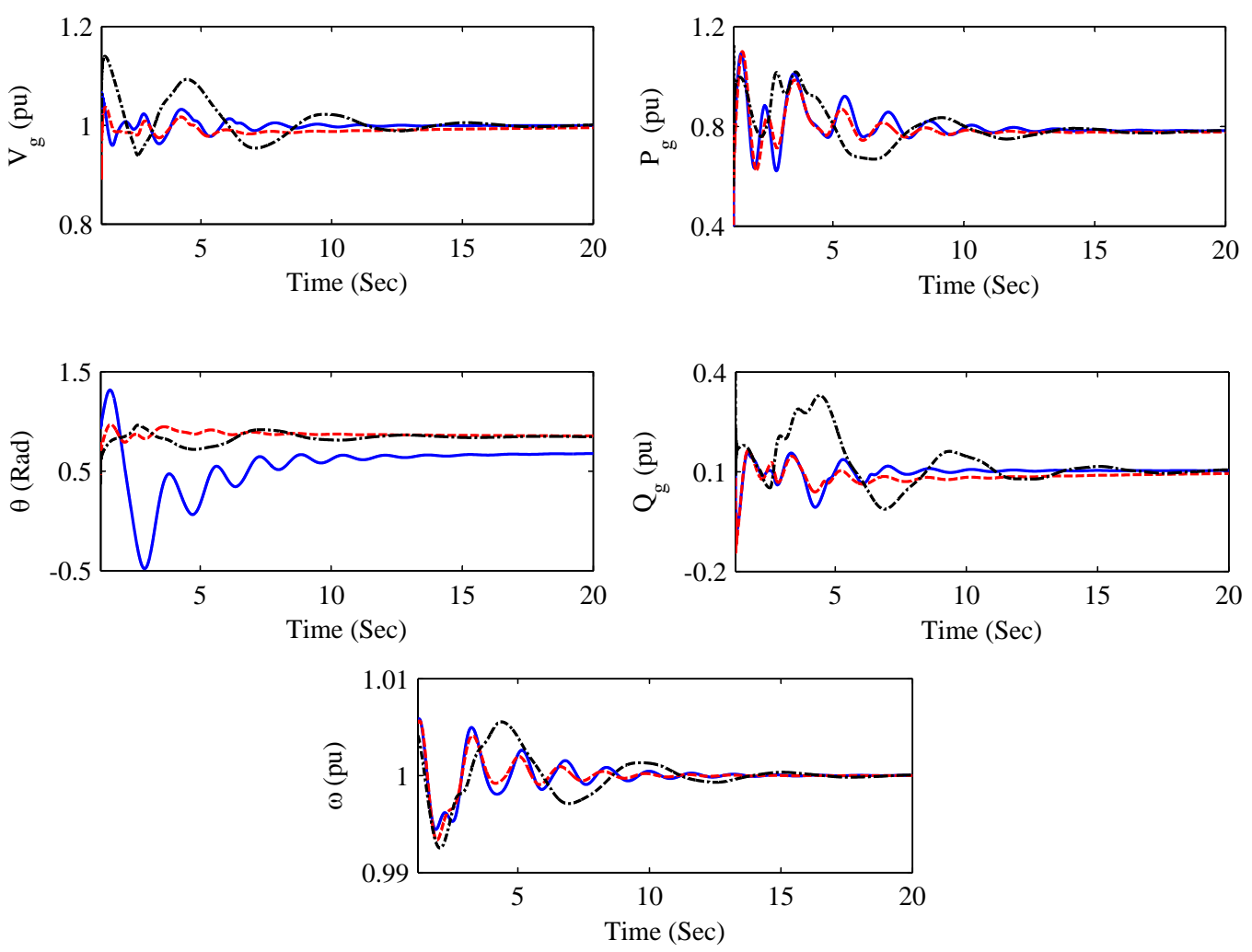

Figure 3: Three sets of Matlab/SimPowerSystems simulation generated PMU measured data. Blue solid lines: Set 1; Read dashed lines: Set 2; Black dot-Dashed: Set 3. 

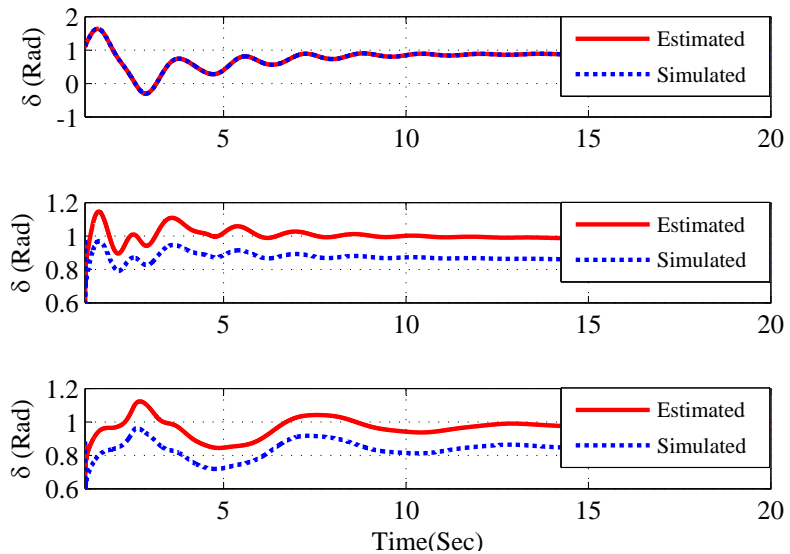

Figure 4: Rotor angle estimation and simulation results. Top: Set 1, Middle: Set 2, Bottom: Set 3
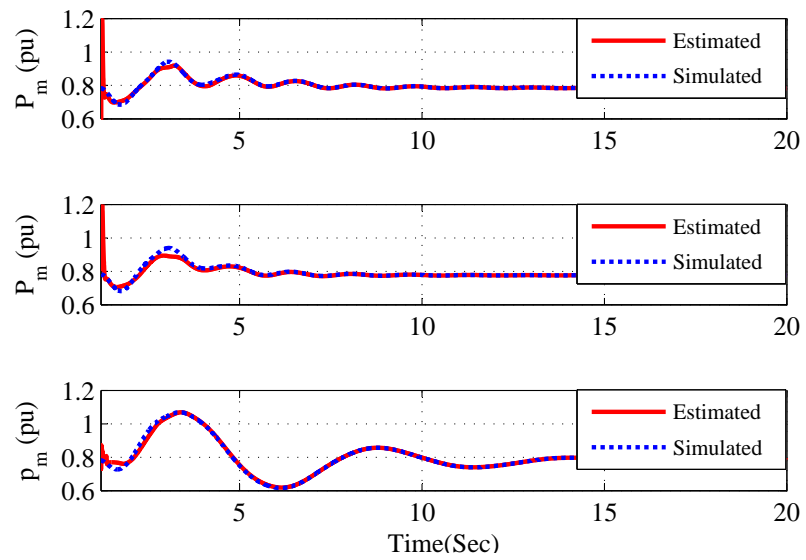

Figure 6: Mechanical Power estimation and simulation results. Top: Set 1, Middle: Set 2, Bottom: Set 3.
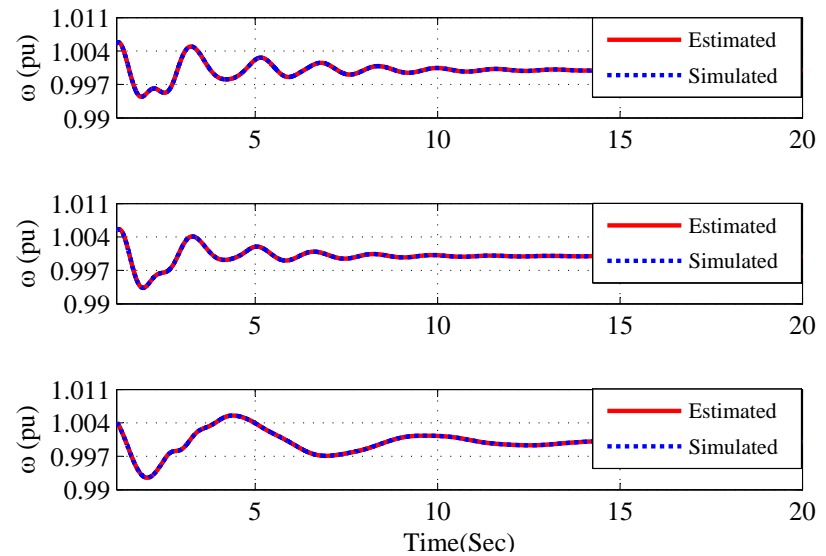

Figure 5: Rotor Speed estimation and simulation results. Top: Set 1, Middle: Set 2, Bottom: Set 3.
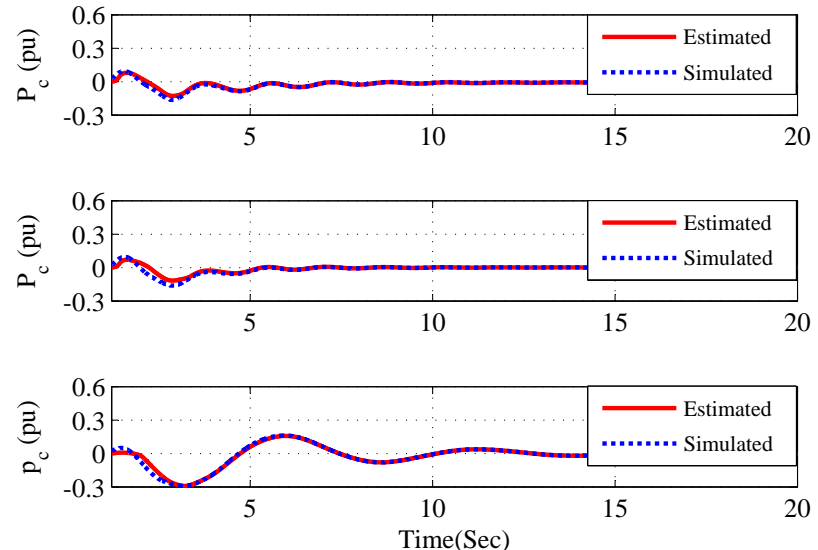

Figure 7: Mechanical Power reference point estimation and simulation results. Top: Set 1, Middle: Set 2, Bottom: Set 3 .
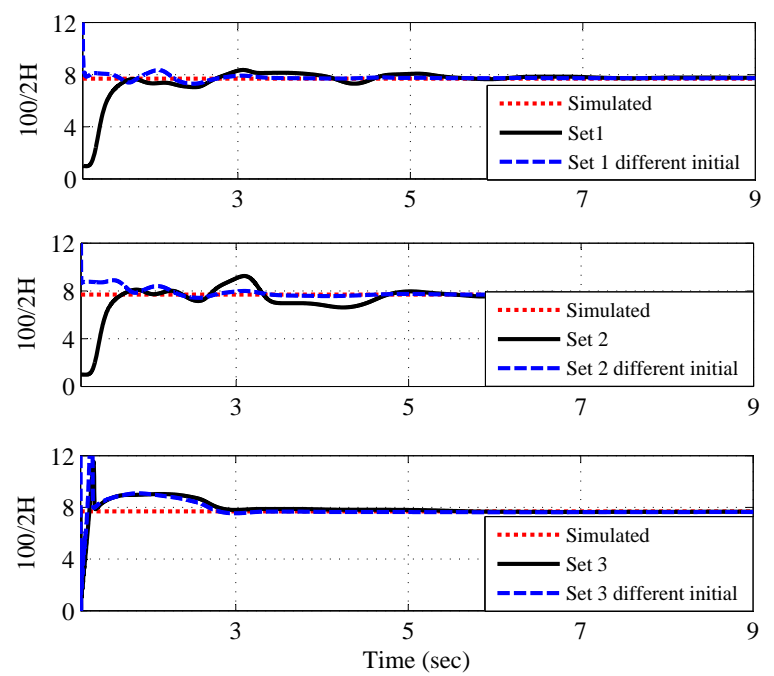

Figure 8: Inertia constant estimation and simulation results. Top: Set 1, Middle: Set 2, Bottom: Set 3. 

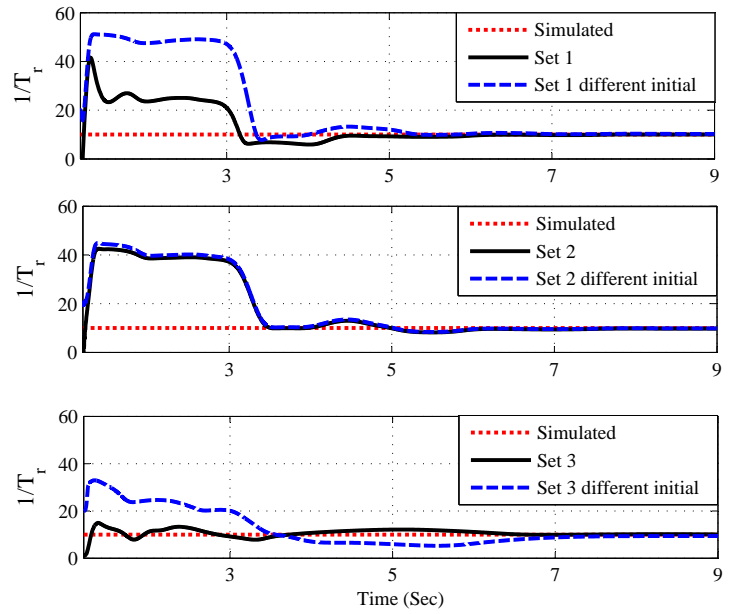

Figure 9: Turbine time constant estimation and simulation results. Top: Set 1, Middle: Set 2, Bottom: Set
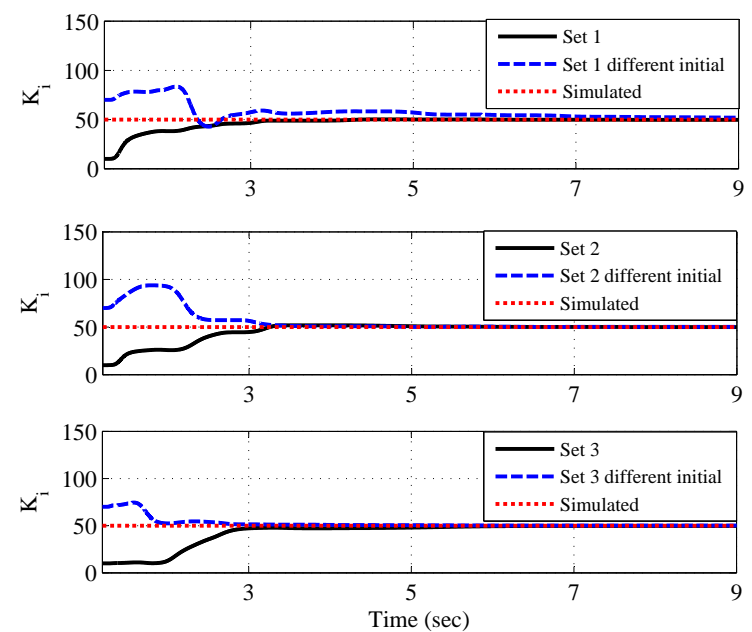

Figure 11: Secondary Frequency Control Integrator Gain estimation and simulation results. Top: Set 1, Middle: Set 2, Bottom: Set 3.
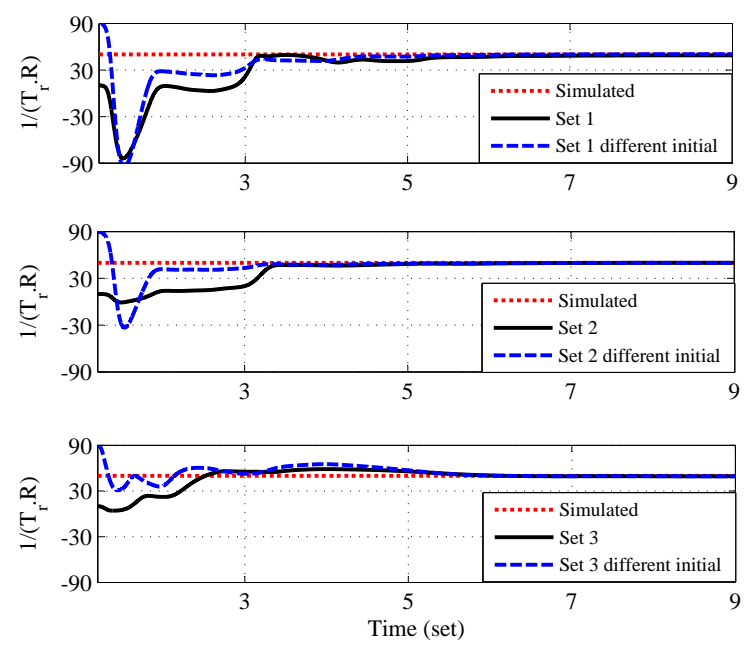

Figure 10: Droop regulation estimation and simulation results. Top: Set 1, Middle: Set 2, Bottom: Set 3.
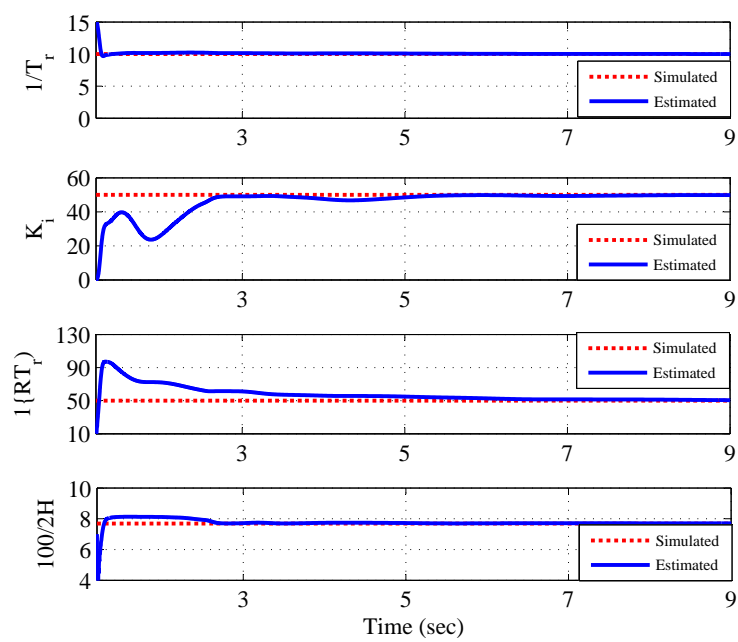

Figure 12: Set 3 All Generator Parameters estimation and simulation results.
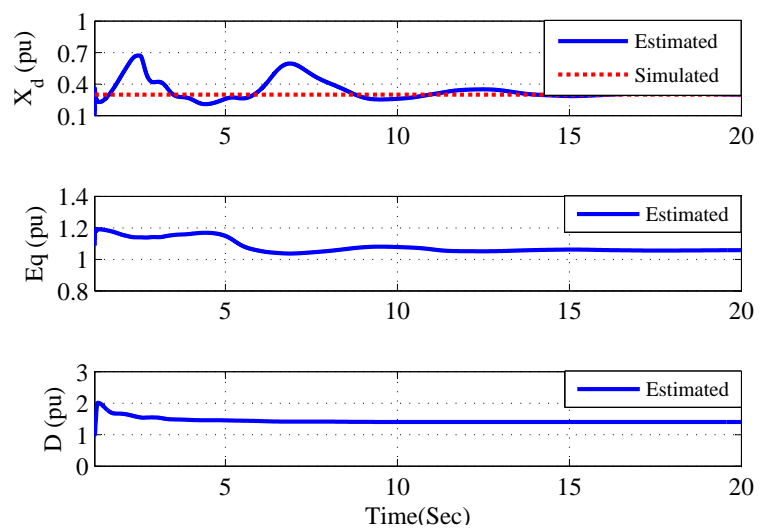

Figure 13: Set 3 All Generator Parameters estimation and simulation results. 

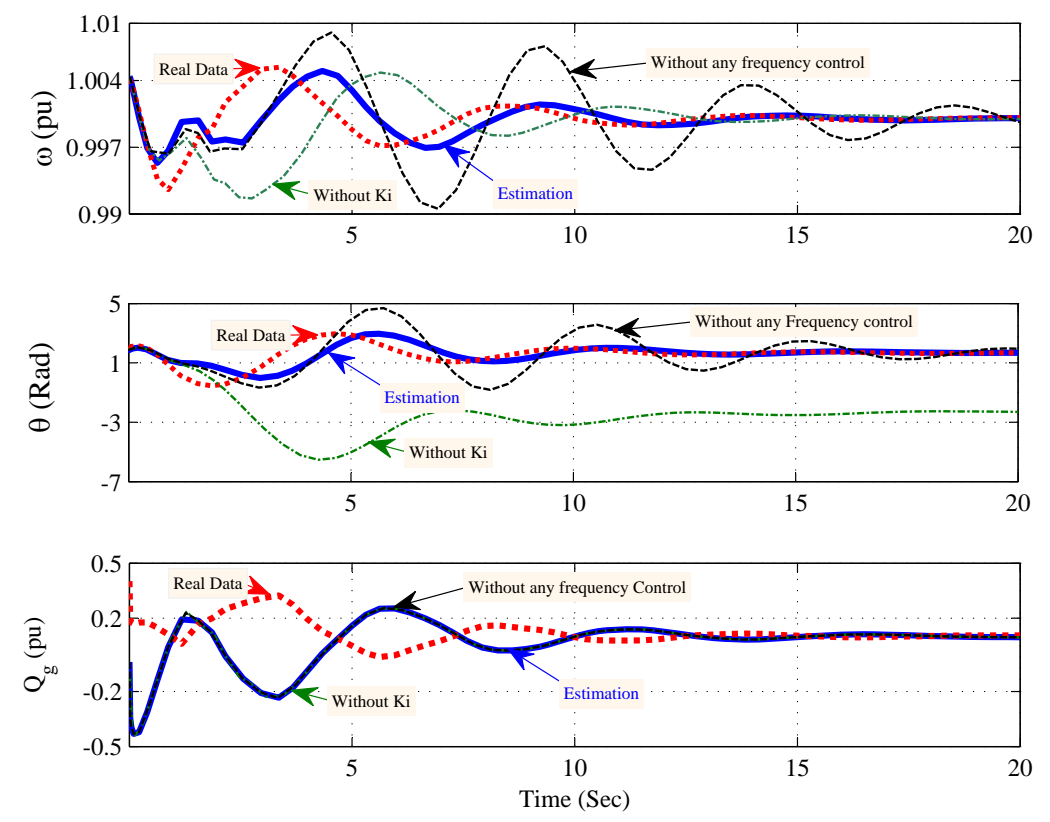

Figure 14: Set 3 Model validation and outputs comparison. Top: Rotor Speed $\omega$, Middle: Voltage Phase Angle $\theta$, Bottom: Reactive Power $Q_{g}$
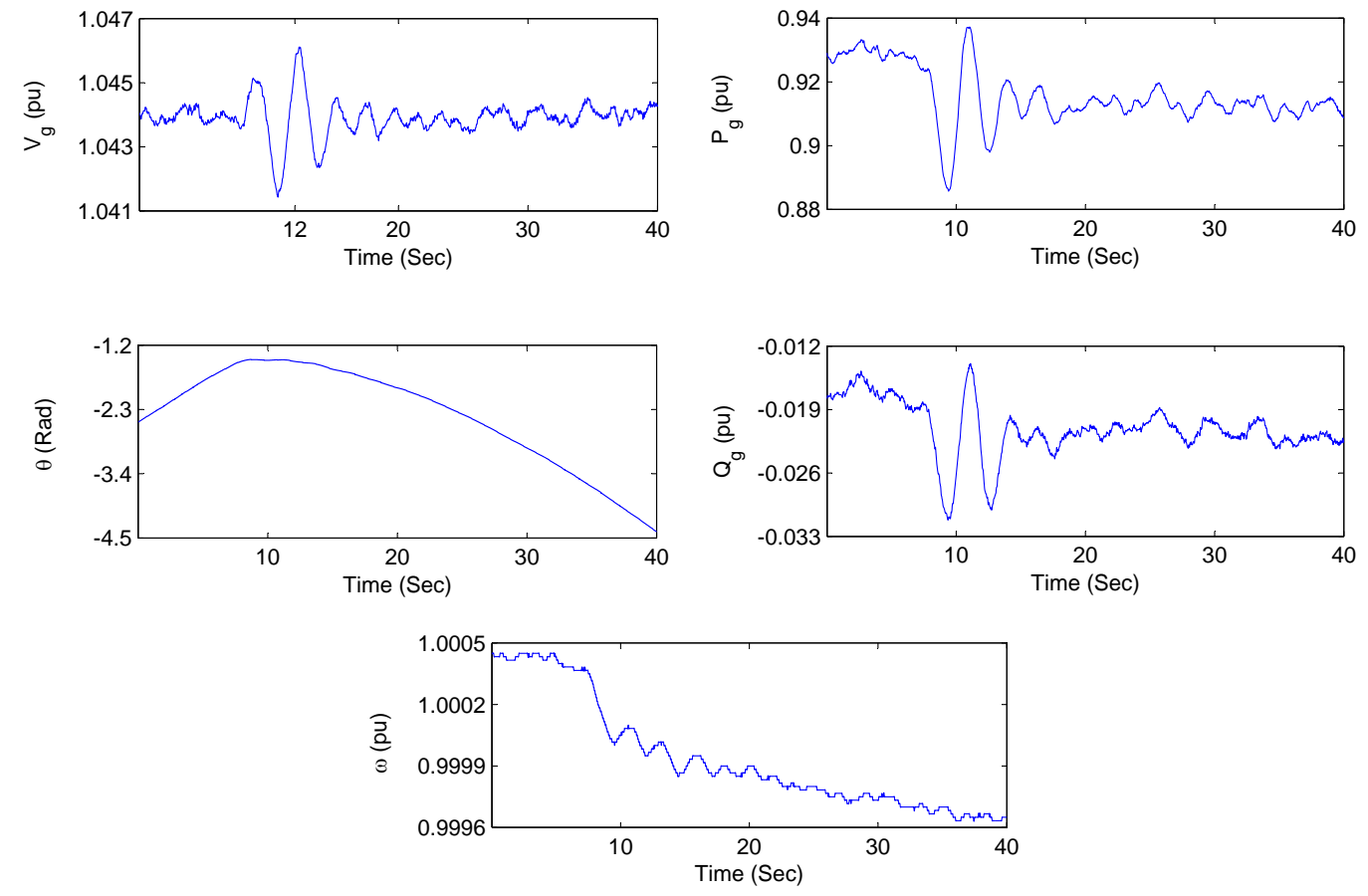

Figure 15: Real-world PMU data obtained from a PMU installed at a $500 \mathrm{kV}$ substation. The data has been scaled to pu values, where $S_{b}=1000 \mathrm{MW}, V_{b}=500 \mathrm{kV}$. 

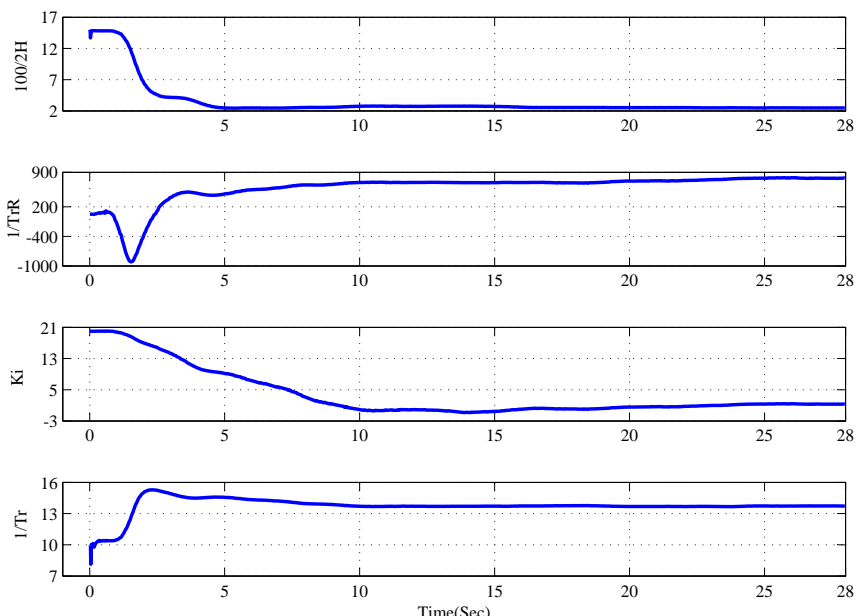

Figure 16: Real-world system parameter estimation results.
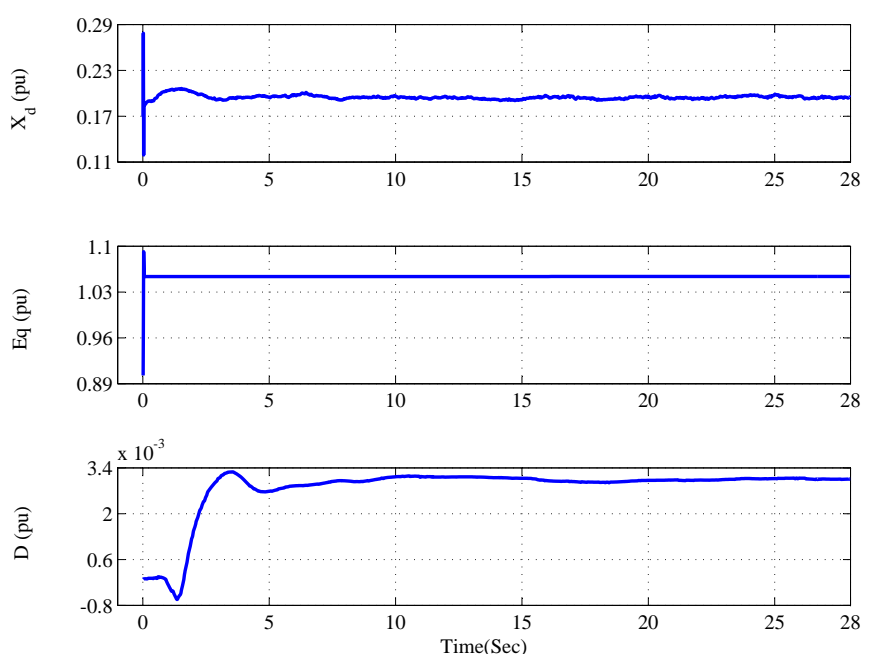

Figure 17: Real-world parameter estimation results. Top: $x_{d}^{\prime}$, Middle: $E_{q}$ Estimation, Bottom: Damping Estimation.
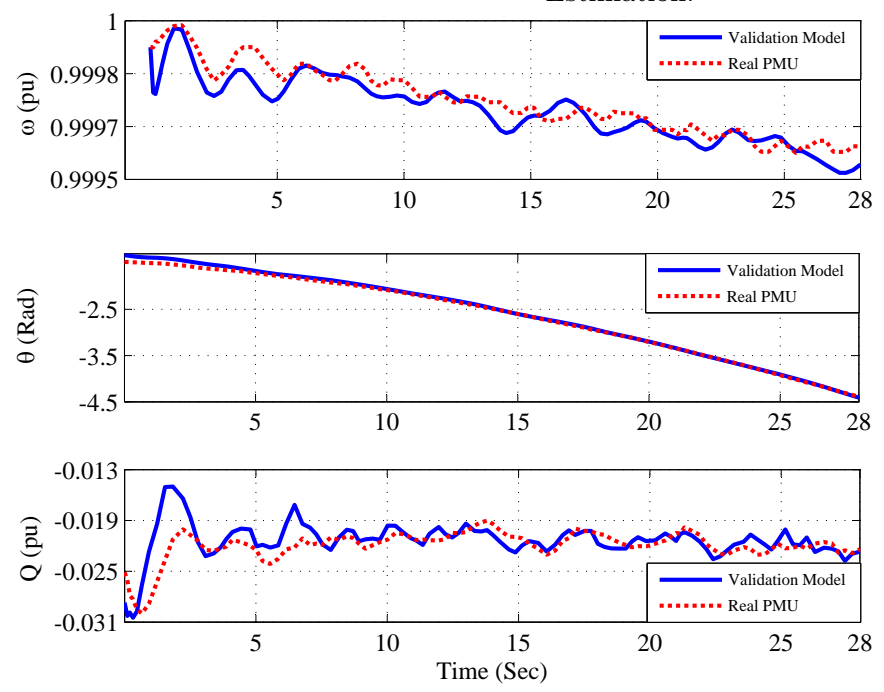

Figure 18: Comparison of the real-world measurements and the simplified simulation model outputs. Top: Rotor Speed $\omega$, Mid: Voltage Phase Angle $\theta$, Bottom: Reactive Power $Q_{g}$ 